which have been developed mainly under the form of substitution groups.

It may be well to add that the symbol $s t s^{-1} t^{-1}$ has been used in substitution groups for a long time, but its use has been very limited. As far as we know its practical application to determine important properties of a group was first explained in the recent article in the Quarterly Journal to which we referred above.

GöTTINGEN,

September, 1896.

\title{
NUMERICALLY REGULAR RETICULATIONS UPON SURFACES OF DEFICIENCY HIGHER THAN 1.
}

BY PROFESSOR HENRY S. WHITE.

By the term reticulation I shall designate for present purposes any system of lines lying upon a closed surface, together with all the points in which these lines intersect one another. Further I shall assume that they divide the surface into portions, of which each by itself is simply connected, $i$. e., has deficiency zero. These portions of the closed surface may be termed faces, and their intersection points vertices, while each boundary line terminated by two consecutive vertices is an edge. If $F, V$ and $E$ denote the numbers of faces, vertices and edges, respectively, in a reticulation, and $p$ the deficiency of the supporting surface, then Euler's relation for convex polyedra, generalized, will be

$$
E=V+F+2 p-2 \text {. }
$$

A reticulation is clearly entitled to be called numerically regular when it has:

1. In every vertex a constant number of termini of edges; call this number $\rho+2=r$.

2. In every circuit bounding a face a constant number of edges, call this number $\sigma+2=s$.

We may for the present regard these two numbers $\rho$ and $\sigma$ alone as characteristics of a regular reticulation; there will remain for subsequent inquiry the determination of the number of essentially different types having any given set of characteristics $\rho, \sigma$, and $p$. From these three the values of $F, V$, and $E$ can be computed, as will be seen below. Counting then as one class all regular reticulations characterized by the same values of $\rho$ and $\sigma$, it can be shown that on a surface of given deficiency $p$, there can exist only a finite number of classes of numerically regular reticulations. 
To prove this we notice that the edges may be enumerated in two ways, either by counting the vertices or by counting the faces:

$$
\begin{aligned}
& 2 E=V r=V(\rho+2), \\
& 2 E=F s=F(\sigma+2) .
\end{aligned}
$$

Combining these equations with Euler's relation and eliminating two of the three letters $E, V, F$, we find expressions for the values of these three quantities in terms of $\rho, \sigma$ and $p$.

$$
\begin{gathered}
F=\frac{(4 p-4)(\rho+2),}{\rho \sigma-4}, \quad V=\frac{(4 p-4)(\sigma+2)}{\rho \sigma-4}, \\
E=\frac{(2 p-2)(\rho+2)(\sigma+2)}{\rho \sigma-4} .
\end{gathered}
$$

The restriction of $p$ to values greater than unity makes all three numerators positive integers. The three cases where the denominator is zero are common to all deficiencies, being the division of a surface into triangles, quadrilaterals and hexagons respectively; $E, F$ and $V$ being then infinite except for $p=1$, which particular value makes them indeterminate. Omitting these cases, we see that positive values of $F, V$ and $E$ require positive values of $\rho \sigma-4$. Accordingly $\rho \sigma$ has the lower limit 5, and we seek next to find an upper limit.

Two cases may be distinguished. Either $\rho=\sigma$, or else they are unequal. Since the formulæ for $V$ and $F$ are merely exchanged when $\rho$ and $\sigma$ are permuted, we shall assume in the second case that $\rho$ is the greater.

From $\rho=\sigma$ it follows that

$$
F=V=\frac{4 p-4}{\sigma-2}
$$

$i$. e., $\sigma-2$ can have only those values that are factors of $4 p-4$, a finite number, and the upper limit of $\rho \sigma$ is for this case $(4 p-2)^{2}$. From $\rho>\sigma$ it follows also that $\rho \sigma<$ $(4 p-2)^{2}$. For since $\frac{V}{F}=\frac{\sigma+2}{\rho+2}$, we have $F>V$ and

$$
\begin{aligned}
& F-V=\frac{(4 p-4)(\rho-\sigma)}{\rho \sigma-4} \geqq 1, \\
& (4 p-4)(\rho-\sigma)-\rho \sigma \geqq-4,
\end{aligned}
$$


adding $(4 p-4)^{2}$ to each side, and factoring we have

$$
(4 p-4+\rho)(4 p-4-\sigma) \geqq 4(2 p-1)(2 p-3) .
$$

Since $p>1$, the second member is positive, therefore

$$
\begin{gathered}
4 p-4-\sigma>0 \\
\sigma<4 p-4
\end{gathered}
$$

As $V \geqq 1, \rho \sigma-4 \equiv(4 p-4)(\sigma+2)$,

or $\quad \rho \sigma<4\left(4 p^{2}-6 p+3\right)$,

an upper limit for the second case, less than $(4 p-2)^{2}$.

Since then $\rho \sigma$ has for every value of $p>1$ finite lower and upper limits, and since any admissible value has only a finite number of pairs of factors, and only those values of $\rho$ and $\sigma$ are to be retained which give integral values to $V$ and $F$, it is now shown that for given $p$ only a finite number of classes of regular reticulations can exist.

In tabulating the classes belonging to a given $p$, it is convenient to treat as one any two which differ only in that the values of $\rho$ and $\sigma$, hence also of $V$ and $F$, are permuted. In the table for $p=2$, we find 14 classes, representing 25

\begin{tabular}{|c|c|c|c|c|c|c|}
\hline No. & $\rho \sigma$ & $\rho$ & $\sigma$ & $V_{r}$ & $F_{s}$ & $E$ \\
\hline $\begin{array}{r}1 \\
2 \\
3 \\
4 \\
5 \\
6 \\
7 \\
8 \\
9 \\
10 \\
11 \\
12 \\
13 \\
14\end{array}$ & $\begin{array}{r}5 \\
6 \\
6 \\
7 \\
8 \\
8 \\
9 \\
10 \\
12 \\
16 \\
16 \\
20 \\
24 \\
36\end{array}$ & $\begin{array}{r}5 \\
6 \\
3 \\
7 \\
8 \\
4 \\
3 \\
10 \\
6 \\
16 \\
4 \\
10 \\
8 \\
6\end{array}$ & $\begin{array}{l}1 \\
1 \\
2 \\
1 \\
1 \\
2 \\
3 \\
1 \\
2 \\
1 \\
4 \\
2 \\
3 \\
6\end{array}$ & $\begin{array}{c}12_{7} \\
6_{8} \\
8_{5} \\
4_{9} \\
3_{10} \\
4_{6} \\
4_{5} \\
2_{12} \\
2_{8} \\
1_{18} \\
2_{6} \\
1_{12} \\
1_{10}\end{array}$ & $\begin{array}{r}28_{3} \\
16_{3} \\
10_{4} \\
12_{3} \\
10_{3} \\
6_{4} \\
4_{5} \\
8_{3} \\
4_{4} \\
6_{3} \\
2_{6} \\
3_{4} \\
2_{5} \\
1_{8}\end{array}$ & $\begin{array}{r}42 \\
24 \\
20 \\
18 \\
15 \\
12 \\
10 \\
12 \\
8 \\
9 \\
6 \\
6 \\
5 \\
4\end{array}$ \\
\hline
\end{tabular}
when such dual reticulations are distinguished.

Regular Reticulations for $p=2$.

For realizing those number-schemes upon models, I have not formulated any a priori infallible method, but have treated the problem as a test exercise for the geometric im- 
agination. My own success was limited to the production of two models. The other twelve, with one exception, were designed with great facility and neatness by Mr. OLIN H. BAsquin, Fellow in Physics in Northwestern University. Any closed surface free from double lines and possessing visibly the deficiency 2 is suitable for displaying graphically these 14 (or 25) reticulations. Five have been illustrated upon small plaster forms cast for the purpose, double rings; and the entire series is traced upon flat cards through which two apertures have been made. Both sides of a card are considered as forming the surface, the opposite sides being connected by every apparent sharp edge. In only one instance did Mr. Basquin find it necessary to let the same line occur twice in the boundary of a single face; but often it is seen that the same point occurs twice or even thrice in the boundary of the same face. As an experimental fact it is worth while to record that every class of numerically regular reticulations that is arithmetically possible upon a surface of deficiency $p=2$ has been realized graphically in these 14 models, classes dual to each other being counted as one.

The 14 regular reticulations of the above scheme for $p=2$ are fundamental to certain others that exist on surfaces of every deficiency $p>2$. From each of the former is derived one of the latter for each deficiency. Such a derivation is arithmetically evident, and Mr. Basquin has proposed a practical method for following out in the model the arithmetical indications. If I illustrate this method by derivation from $p=2$ to $p=3$, the extension of the process to $p=4,5$, etc., will be immediately evident; and the similar derivation from $p=3$ to $p=5$, 7, etc., or from $p=n+1$ to $p=2 n+1,3 n+1$, etc., will need no explanation.

If on a surface of deficiency 2 a regular reticulation is characterized by the numbers $\rho^{\prime}, \sigma^{\prime}, V^{\prime}, F^{\prime}, E^{\prime}$, then on a surface of deficiency 3 there is a regular reticulation marked by the same $\rho^{\prime}, \sigma^{\prime}$, together with values $V, F, E$, such that

$$
V=2 V^{\prime}, \quad F=2 F^{\prime}, \quad E=2 E^{\prime} .
$$

For if $\mu^{\prime}, \sigma^{\prime}$ render $V^{\prime}, F^{\prime \prime}, E^{\prime}$ integers, where

$$
V^{\prime}=\frac{4\left(\rho^{\prime}+2\right)}{\rho^{\prime} \sigma^{\prime}-4}, \quad F^{\prime}=\frac{4\left(\rho^{\prime}+2\right)}{\rho^{\prime} \sigma^{\prime}-4}, \quad E^{\prime}=\frac{2\left(\rho^{\prime}+2\right)\left(\sigma^{\prime}+2\right)}{\rho^{\prime} \sigma^{\prime}-4},
$$

then they render integral also the values $V, F, E$ belonging to $p=3,(4 p-4=8)$, where 


$$
\begin{gathered}
V=\frac{8\left(\sigma^{\prime}+2\right)}{\rho^{\prime} \sigma^{\prime}-4}=2 V^{\prime}, \quad F=\frac{8\left(\rho^{\prime}+2\right)}{\rho^{\prime} \sigma^{\prime}-4}=2 F^{\prime} \\
E=\frac{4\left(\rho^{\prime}+2\right)\left(\sigma^{\prime}+2\right)}{\rho^{\prime} \sigma^{\prime}-4}=2 E^{\prime}
\end{gathered}
$$

All such sets of numbers $V, F, E$, obtained by doubling the numbers in the table for $p=2$, I shall call derivative sets for $p=3$; the corresponding reticulations will also be called derivative. All others I shall call special sets or reticulations for $p=3$. For $p=5$ there would be of course two kinds of derivatives; those coming from the scheme for $p=2$ by five-folding the numbers in the columns $V, F, E$, and those coming from the special sets for $p=3$ by doubling the members $V, F, E$. So for higher deficiencies there would be often several kinds of derivatives, but for a prime $(p-1)$ only one kind.

For $p=3$ the special regular reticulations are readily determined by trial; for the value of $(\rho \sigma-4)$ must contain at least the factor 8 , with either $\rho$ or $\sigma$ an odd number, otherwise it will contain a factor 16 .

\begin{tabular}{|c|c|c|c|c|c|c|}
\hline No. & $\rho \sigma$ & $\rho$ & $\sigma$ & $V_{r}$ & $F_{s}$ & $E$ \\
\hline $\begin{array}{l}1 \\
2 \\
3 \\
4 \\
5 \\
6 \\
7\end{array}$ & $\begin{array}{r}12 \\
12 \\
28 \\
28 \\
36 \\
60 \\
100\end{array}$ & $\begin{array}{r}4 \\
12 \\
7 \\
28 \\
18 \\
12 \\
10\end{array}$ & $\begin{array}{r}3 \\
1 \\
4 \\
1 \\
2 \\
5 \\
10\end{array}$ & $\begin{array}{l}\mathbf{5}_{6} \\
3_{14} \\
2_{9} \\
\mathbf{1}_{30} \\
\mathbf{1}_{20} \\
\mathbf{1}_{14} \\
\mathbf{1}_{12}\end{array}$ & $\begin{array}{r}6_{5} \\
14_{3} \\
3_{6} \\
10_{3} \\
5_{4} \\
2_{7} \\
1_{12}\end{array}$ & $\begin{array}{r}15 \\
21 \\
9 \\
15 \\
10 \\
7 \\
6\end{array}$ \\
\hline
\end{tabular}

Special Regular Reticulations for $p=3$.

For these seven special classes Mr. Basquin has constructed card models. Here, therefore, we record, as an empirical fact, that every special class of regular reticulations that is arithmetically possible upon a surface of deficiency 3 can be realized graphically.

How to produce a derivative reticulation from its fundamental remains to be explained. With a model of some one reticulation of deficiency 2 before the eye, prepare or imagine a duplicate of it; and let both be of material easily cut and distorted. Cut the first along any closed line that does not divide it into separate parts. Make an exactly similar cut in the second model. Distort each severed ex- 
tremity through $90^{\circ}$ in such a direction that then the models can be opposed to each other and each severed portion can be united to the duplicate of that from which it was divided. The resulting surface will be of deficiency 3 and will contain a regular reticulation with twice as many vertices, faces and edges as its original. As illustrations of this mode of derivation Mr. Basquin has found fairly simple examples to arise from Nos. 3, 6, 9, 11 of the table for deficiency 2 . It will be seen that the closed line marking the cut may be equally well any number, not greater than $p-1$, of non-intersecting closed curves. Where $p>2$, this observation points to an interesting variety of derivative reticulations.

Of the starred polyedra, one is found to belong to this scheme of regular reticulations, namely the starred dodecædron; in the others, the connectivity of the individual faces and vertices has to be taken into account. Semi-regular reticulations, analogous to the solids of Archimedes, I have not investigated; but I should expect that the graphical production of such would give the inventive faculties more exercise and pleasure than the construction of those wholly regular.*

NORTHWESTERN UNIVERSITY,

Evanston, ILL., August, 1896.

\section{CORRECTION.}

The theorem given in the first paragraph of the article "Note on the Special Linear Homogeneous Group," p. 336, of the last volume of the Bulletin, is not true, and is not, as there stated, a consequence of results given on p. 232. The theorem given in the second paragraph, p. 336, regarding the special linear homogeneous group in $n$ variables, for $n=2$ or composite, and the method of proof which follows, holds also if $n$ is an odd prime. Henry Taber.

* The theory of canonical dissections of a Riemann surface leads to the completion of the foregoing discussions. - H. S. W. 